\title{
La estrella de Belén en el Evangelio de Mateo, en el Protoevangelio de Santiago y en otros tres textos apócrifos: un análisis literario y comparativo
}

\author{
The star of Bethlehem in the Gospel of Matthew, in the Protevangelium of \\ James and in other three apocryphal texts: \\ a literary and comparative analysis
}

\begin{abstract}
Resumen
Este artículo explora el rol literario de la estrella de Belén del Evangelio de Mateo en cuatro textos apócrifos. Algunos aspectos literarios de los textos apócrifos son examinados. Este examen inicialmente determina las relaciones editoriales que existen entre estos y el texto canónico de Mateo. El estudio comienza con el Protoevangelio de Santiago, el cual edita y emplea con creatividad el Mateo canónico en lo tocante al relato de los magos y la estrella. Seguidamente, el artículo examina la actividad editorial que tres apócrifos realizan del Protoevangelio, en relación al astro. Estos son el Evangelio Árabe de la Infancia, el Evangelio Armenio de la Infancia, y el Evangelio de Seudo-Mateo. Un examen comparativo de los cuatro, permite concluir que, si bien existen elementos editoriales, el motivo de la estrella comprende un cuadro semejante presentado por el Mateo canónico, delineando su actuar en términos de movimiento.
\end{abstract}

Palabras clave: Estrella de Belén. Evangelio de Mateo. Evangelios Apócrifos. Protoevangelio de Santiago. Evangelio Árabe de la Infancia. Evangelio Armenio de la Infancia. Evangelio de Seudo-Mateo.

\begin{abstract}
This article explores the literary role of the Star of Bethlehem of Matthew's Gospel in four apocryphal texts. Some literary aspects of the apocryphal texts are examined. This initial analysis determines the existence of editorial connections that reveal their relationship with the canonical text of Matthew. It begins by studying the Protevangelium of James, which creatively edits and uses the canonical Matthew regarding the story of the magi and the star. Then, the article examines the editorial activity that three apocryphal texts make of the Protevangelium concerning the star. These are: The Arabic Infancy Gospel, the Armenian Infancy Gospel and the Pseudo Gospel of Matthew. A comparative examination of these four apocryphal Gospels allows us to understand that even though editorial elements exist, the motive of the star resembles Matthew's Gospel, describing the star in terms of movement.
\end{abstract}

Keywords: Star of Bethlehem. Gospel of Matthew. Apocryphal Gospels. Protevangelium of James. Arabic Infancy Gospel. Armenian Infancy Gospel. Pseudo Gospel of Matthew.

Artigo submetido em 1 de novembro de 2018 e aprovado em 27 de setembro de 2019.

* Doutor em Teologia pela Universidade de Auckland, Nova Zelândia. Professor titular do Centro Universitário Adventista de São Paulo (UNASP). País de origem: Chile. E-mail: carlos.olivares@unasp.edu.br 


\section{Introducción}

El Evangelio de Mateo informa que alrededor del tiempo en que Jesús nace unos magos que venían del oriente le visitan (Mt 2,1-12). Los magos afirman haber visto una estrella, la cual, a medida que la narrativa avanza, los guía hasta un Jesús infante que estaba reunido con sus padres (2,2, 7, 9-10). El astro, conocido popularmente como la estrella de Belén, ha sido estudiado desde diversas ópticas. Estas comprenden, entre otras (BARTHEL; VAN KOOTEN, 2015), lecturas astronómicas (FERRARI-D’OCCHIEPPO, 1989, p. 41-53), midrásicas (VIVIANO, 1996, p. 58-64), patrísticas (HEGEDUS, 2003, p. 81-95), artísticas (DUCHESNEGUILLEMIN, 1985, p. 149-157) e histórico-contextuales (ALLISON, 2005, p. 1741).

Este artículo propone abordar el estudio de la estrella de Belén desde la perspectiva de cuatro evangelios apócrifos. Para lograr esto, primero se examina el rol de la estrella en el Protoevangelio de Santiago (Prot. San.), ponderando el uso creativo que este hace del Mateo canónico. Luego, se analiza el trabajo redaccional del Evangelio Árabe de la Infancia (Ev. Arab. Inf.), el Evangelio Armenio de la Infancia (Ev. Arm. Inf.) y el Evangelio de Seudo-Mateo (Ps.-Mt). La raison d'etre de esta triple elección estriba en que cada uno de estos apócrifos basan su contenido en el Protoevangelio, y por lo tanto denotan una fuente común de interpretación. Esto significa que si bien existen otros textos apócrifos que relatan el episodio de la estrella, estos no son parte de esta unión editorial del cual el Protoevangelio, y los textos ya citados, disfrutan. Por lo tanto, el examen de otros apócrifos ha sido omitido.

Metodológicamente, un análisis de este tipo envuelve considerar la estrella de Belén como un motivo literario. Esto implica que este artículo no busca determinar la validez histórica del astro, o de los personajes mencionados, ni tampoco emitir un juicio de valor respecto a los documentos apócrifos. Su propósito, al contrario, es literario. El que busca establecer diacrónicamente la 
transformación e interpretación del tema de la estrella en los escritos referidos, evaluándolos a partir de la narración seminal de Mateo. Igualmente, a fin de establecer una fecha apropiada para cada apócrifo, así como para determinar su correspondencia redaccional, este artículo primero discute los pormenores histórico-crítico-literarios que cada documento apócrifo en estudio exhibe.

\section{El Protoevangelio de Santiago}

La composición del Protoevangelio de Santiago, al menos en su forma primitiva, ha sido fechada alrededor del siglo segundo (EHRMAN; PLEŠE, 2011, p. 35; CULLMANN, 2003, p. 423). El comentario al Evangelio de Mateo escrito por Orígenes (Comm. Matt. 10.17), y posiblemente la obra Stromata, de Clemente de Alejandría (Str. 7.16.93), dos textos compuestos entre los siglos segundo y tercero, concuerdan sobre la existencia temprana del apócrifo (GONZÁLEZ et al., 1997, p. 75). Igualmente, el Papiro Bodmer V, cuya fecha de composición ronda el tercer siglo (ZERVOS, 2012, p. 177-194), apunta a que el Protoevangelio pudo haber sido escrito una centuria antes (FOSTER, 2007, p. 575; FOSTER, 2008, p. 113).

En términos de la recepción del apócrifo, la ausencia de testigos tempranos en la región occidental del imperio parece sugerir que el tal tuvo una aceptación mayor en oriente (SANTOS OTERO, 2005, p. 58; EHRMAN; PLEŠE, 2011, p. 31). Sin embargo, homilías en latín escritas en un período posterior (DE ALDAMA, 1962, 1975; KAESTLI, 2001), así como adaptaciones literarias que luego conformarían la redacción de otros escritos apócrifos (SCHRÖTER, 2015, p. 175176), revelan que el Protoevangelio ganó paulatinamente terreno en occidente.

La influencia del apócrifo en ambas regiones del imperio puede sin duda observarse al notar las diferentes traducciones que el texto experimentó en el tiempo (GEERARD, 1992, p. 25-30 [Nr. 51]). Del mismo modo, la preponderancia del tal emerge en las diversas obras artísticas antiguas que basaron sus representaciones en las escenas descritas en el Protoevangelio (CARTLIDGE, 2014; CARTLIDGE; ELLIOTT, 2001, p. 33, 41, 78). 
El texto del apócrifo, quizás compuesto y preservado originalmente en griego (SANTOS OTERO, 2005, p. 57), presenta un sinnúmero de variantes textuales. Sin embargo, el Protoevangelio, aunque con leves diferencias, refleja una historia con parámetros narrativos homogéneos, los que son compartidos por un número importante de manuscritos (COTHENET, 1988, p. 4259).

Un análisis de las fuentes que el Protoevangelio emplea refleja un proceso de redacción que emula, y usa embrionariamente, a los evangelios de Mateo y Lucas (GOODACRE, 2018, p. 57-76). En la misma línea, el apócrifo asimismo evidencia, a medida que la historia es contada, una adaptación midrásica de la Biblia Hebrea (QUARLES, 1998, p. 139-149). Empero, la escena en que los magos visitan a Jesús, y el rol de la estrella en el desarrollo del argumento, comprenden una redacción mateana que sigue de cerca este relato, y que el Protoevangelio claramente edita.

\subsection{La estrella de Belén en el Protoevangelio de Santiago}

El episodio de los magos en el Protoevangelio comienza con José preparándose para ir a Judea (Prot. San. 21.1). El autor del apócrifo identifica Judea con Jerusalén (GONZÁLEZ et al., 1997, p. 128; AMANN, 1910, p. 257-258), a donde José tiene la intención de dirigirse luego que María da a luz (Prot. San. 21.3; cf. 20.4). El motivo del viaje, y en virtud de la narrativa total del apócrifo, sugiere que José y María viven en Jerusalén (GONZÁLEZ et al., 1997, p. 71), y desde ahí parten a Belén para que José sea censado (Prot. San. 17.1). En el camino, no obstante, antes de llegar a Belén (17.2-3), Jesús nace (18.1). Una vez que esto acontece, José pretende volver a su hogar, en tanto unos magos que vienen del oriente aparecen en escena (21.1).

A diferencia del Evangelio de Mateo, en donde los magos llegan a Jerusalén (Mt 2,1-2), en el Protoevangelio arriban a Belén (Prot. San. 21.1). Estos aparecen en el relato preguntado por el rey de los judíos, a quien ellos venían a adorar, afirmando además haber visto su estrella en el oriente (Prot. San. 21.1; Mt 2,1-2). 
Emulando a Mateo, el Protoevangelio informa que Herodes se inquieta, y consulta a los líderes religiosos judíos el lugar en el que nacería el Mesías (Prot. San. 21.2; Mt 2,3-4).

$\mathrm{Al}$ enterarse que las Escrituras indican que la profecía del Cristo se cumpliría en Belén de Judea, Herodes convoca a los magos, aparentemente en Jerusalén, interpelándolos respecto a la señal que habían visto, y que apuntaba al rey nacido. Los magos, apartándose del relato mateano, responden a Herodes haber contemplado una estrella enorme que brillaba entre los astros, eclipsándolos, al punto de hacerlos desaparecer. Los magos comprenden que la estrella significa que un rey había nacido, y esa es la razón de su venida (Prot. San. 21.2; cf. Mt 2,5-7). Como en Mateo, Herodes envía a los Magos con la promesa de que, si ellos lo encuentran, él también irá a adorarlo (Prot. San. 21.2; Mt 2, 8).

Al salir los magos de la presencia de Herodes, el Protoevangelio indica que la estrella que ellos habían visto en el oriente nuevamente asume su papel de guía, y los encamina al lugar en donde Jesús había nacido (Prot. San. 21.2; Mt 2, 8). En contraposición al Evangelio de Mateo, Jesús no está en una casa (oikia) sino en una cueva (spelaion). Este escenario, de acuerdo al apócrifo, corresponde al lugar en el que María dio a luz, y desde el cual José desea partir a Jerusalén (Prot. San. 18.1; 19.1-3; 21.1-3; Mt 2,10-11). En Mateo, no obstante, Jesús, quien es un niño, parece tener un tiempo ya de nacido. A este respecto, pareciera que el Protoevangelio comprime y edita escenas del nacimiento de Jesús tomadas de Lucas (Lc 2,1-7), y las complementa, en una fusión narrativa, con el episodio de los magos en Mateo (Mt 2,1-12).

Remedando a Mateo, el Protoevangelio señala que la estrella va delante de los magos hasta que estos encuentran a Jesús. El astro, afirma el narrador, consiste en el mismo que ellos habían visto en el oriente, y que ahora los conduce desde Jerusalén hacia donde Jesús está (Prot. San. 21.2-3; Mt 2,9). Al describir esto, la estrella asume un rol movible, similar al planteado en Mateo (Mt 2,9). Esto queda en evidencia al notar que la estrella se detiene sobre la entrada o el techo de la 
cueva en donde estaba Jesús (Prot. San. 21.3). En algunas variantes textuales es posible incluso leer que la estrella se posó sobre la cabeza de Jesús (TISCHENDORF, 1853, p. 40; AMANN, 1910, p. 260; EHRMAN; PLEŠE, 2011, p. 66), lo cual envuelve que esta debió entrar en la cueva.

En consecuencia, independientemente de la variante escogida, para el autor del Protoevangelio la estrella no es estática. Al contrario, esta cumple una función de conducción, ayudando a los magos a reconocer que un rey había nacido. Luego, asumiendo el mismo cometido, la estrella los conduce al lugar del nacimiento de Jesús. El curso que esta toma en el cielo comprende dirección, el que ocurre en las alturas, y que luego desciende al nivel de los hombres. Esto, porque el movimiento de la estrella no sólo ocurre en el firmamento, sino además aparece debajo de este, posándose sobre la cueva/niño. Otra característica de esta estrella es que ella excede a otros astros en relación a su tamaño y fulgor. El Protoevangelio la describe como un astro enorme, y con un brillo comparativamente superior al que emana de los otros cuerpos celestes.

\section{El Evangelio Árabe de la Infancia}

La composición del Evangelio Árabe de la Infancia, al menos en su arquetipo primitivo, ocurrió posiblemente entre los siglos quinto y sexto (ELLIOTT, 1993, p. 100; BOCKMUEHL, 2017, p. 82). La razón para establecer estas fechas estriba en ciertos detalles que surgen desde el contenido del apócrifo, y en su relación con otras fuentes. El Corán, por ejemplo, retrata a un Jesús todavía bebe hablando desde la cuna con su madre (Q María 19:29-34). ${ }^{1}$ El Evangelio Árabe, por su parte, registra una escena en esencia similar (Ev. Arab. Inf. 1.2). ${ }^{2}$ Debido a este paralelo literario, se ha sugerido que el Corán empleó el contenido del apócrifo (HORN, 2006, p. 136), o de otros escritos semejantes (HORN, 2007, 2008), lo cual ha llevado a concluir que el Evangelio Árabe tiene que haberse escrito antes del surgimiento del islam (JÖRG, 2015, p. 28).

\footnotetext{
${ }^{1}$ Ver El Corán (2007, p. 385).

${ }^{2}$ Ver Santos Otero (2005, p. 139).
} 
Lógicamente el empleo de una fuente también puede ocurrir en términos opuestos, lo cual implica que el Evangelio Árabe pudo haber usado el texto coránico, y no viceversa. Empero, y sin entrar en detalles respecto al posible uso de otras fuentes apócrifas cristianas por parte del Corán (GRYPEOU, 2005, p. 311-316; HORN, 2006, p. 113-150), la antigüedad del relato probablemente encuentra sustento, y por ende una datación preislámica, al establecer un hipotético origen sirio.

En relación a este último caso, se ha postulado que el Evangelio Árabe de la Infancia fue escrito originalmente en siríaco (PEETERS, 1914, p. vii). Evidencias que respaldan esta noción tienen que ver mayormente con las similitudes que este tiene con leyendas originadas en esta lengua respecto a María, la madre de Jesús (PEETERS, 1914, p. xiv-xxix; BUDGE, 1922). Al mismo tiempo, existen trazos de vocablos sirios en el texto, aduciendo un patrón lingüístico que reconoce un pasado no árabe. Tales indicios pueden incluso interpretarse como el de un autor que, aunque posee los rasgos étnicos sirios, quizás escribió el texto en árabe (COWPER, 1867, p. lxxvii). En cualquier caso, a partir de esta relación de dependencia, el evangelio apócrifo en su matriz primigenia, tendría un origen sirio que habría sido preservado en árabe (PEETERS, 1914, p. vi-vii; ELLIOTT, 1993, p. 100; SANTOS OTERO, 2005, p. 137-138; JÖRG, 2015, p. 28).

El texto del apócrifo que actualmente se dispone es precisamente una versión en árabe publicada por Henricus Sike, la que él basó en un manuscrito que hoy ya no existe (GERO, 1971, p. 52; CULLMANN, 2003, p. 457). Esta versión, editada en árabe y latín (SIKE, 1697), ${ }^{3}$ sirvió de base para el texto bilingüe de Thilo (1832, p. 63-158), quien posteriormente editó y corrigió la versión de Sike (PEETERS, 1923, p. 132-133;). Esta última obra fue luego usada por Tischendorf (1853, p. 171-202) en su Evangelia Apocrypha, de la que a su vez se han

${ }^{3}$ El texto en árabe ha sido también reproducido por Thilo (1832, p. 63-158) y Giles (1852, p. 12-32). 
beneficiado un número importante de traducciones modernas (SANTOS OTERO, 2005, p. 137-165; ELLIOTT, 1993, p. 101-107).4

La historia textual del Evangelio Árabe, luego de la versión de Sike, la cual constituye una versión breve del relato apócrifo (VOICU, 2002, p. 14), ha recibido algunas actualizaciones a través del tiempo (PEETERS, 1923; GERO, 1971, p. 52). Una de estas es el manuscrito Laurenziano orientale (n. 387), publicado por Mario Provera, el que ofrece una lectura más extensa y de calidad superior, toda vez que concuerda en reiterados casos con el texto sirio de las historias de la virgen (VOICU, 2002, p. 14-15). Sin embargo, respecto al episodio de los magos y la estrella, las variantes textuales, aunque claramente existentes, aun retratan, como se verá más abajo, un cuadro narrativo similar. 5

Desde el punto de vista de la redacción del Evangelio Árabe de la Infancia, éste emplea en su composición el Protoevangelio de Santiago, el Evangelio de la infancia de Tomás, y otras leyendas apócrifas (ELLIOTT, 1993, p. 100; PEETERS, 1914, p. xiv-xxix). Asimismo, el Evangelio Árabe usa creativamente los textos canónicos de Mateo y Lucas. En particular, las escenas en las que se describe la niñez de Jesús, incluyendo pormenores que son propios de este texto apócrifo (SANTOS OTERO, 2005, p. 137).

A partir de estas fuentes, el Evangelio Árabe puede dividirse en al menos tres partes. En la primera, esto es entre los capítulos 1 al 9, el apócrifo adapta al Protoevangelio de Santiago, así como a Mateo y Lucas. En la segunda, en los capítulos 10 al 35, el Evangelio Árabe utiliza textos míticos orientales, probablemente de origen egipcio (ELLIOTT, 1993, p. 100). En la tercera, desde el capítulo 36 al 55, este se sirve del Evangelio de la Infancia de Tomás (TISCHENDORF, 1853, p. xlix-l; GEERARD, 1992, p. 40).

\footnotetext{
${ }^{4}$ La traducción de Elliott (1993, p. 104-107), no obstante, es parcial, cubriendo solo los capítulos 13 al 40. La de Santos Otero (2005, p. 139-165), con todo, cubre todos los capítulos del texto de Tischendorf.

${ }^{5}$ Para esto ver Sike (1697), Tischendorf (1853, p. 171-202), Voicu (2002), Santos Otero (2005, p. 137-165), Peeters (1914, p. 1-65). Mi lectura del texto, sin embargo, se basa en Tischendorf (1853, p. 171-202) y en Mario Provera. En el caso del texto de Provera, he usado la traducción al italiano de Voicu (2002).
} 
La escena de los magos y la estrella ocurre en la primera parte de esta división, por lo cual el bloque constituye una redacción del Protoevangelio de Santiago. Con todo, incluso asumiendo vínculos narrativos semejantes, el Evangelio Árabe presenta, tanto en su versión breve como larga, detalles particulares a este apócrifo. ${ }^{6}$

\subsection{La estrella de Belén en el Evangelio Árabe de la Infancia}

La versión breve del relato, emulando el evangelio canónico de Mateo, indica que, al nacer Jesús en Belén de Judea durante el reinado de Herodes, unos magos llegan a Jerusalén con presentes de oro, incienso y mirra (Mt 2,1, 11; Ev. Arab. Inf. 7).7 Lo característico del apócrifo, consiste en que su venida acontece como cumplimiento de la predicción de Zaradust. Al adorar a Jesús, y entregarle los regalos, María les regala uno de los pañales del Jesús infante, el que ellos aceptan con honra. ${ }^{8}$

Luego, y sin establecer lapso cronológico alguno, la versión breve del Evangelio Árabe afirma que un ángel aparece delante de los magos, el "que tenía la misma forma de aquella estrella que les había servido de guía en el camino" ( $E v$. Arab. Inf. 7). ${ }^{9}$ Ellos, concluye el apócrifo, siguen el rastro de luz que aparentemente emana del ángel, el que los conduce hasta llegar su tierra.

Por su parte, la versión larga del Evangelio Árabe mantiene las características narrativas descritas en el párrafo anterior. Incluye, con todo, una discusión sobre el número de los magos (Ev. Arab. Inf. 5 [versión larga]). ${ }^{10}$ Inserta también los vacíos informativos dejados en blanco por la versión anterior,

\footnotetext{
${ }^{6}$ Para una lectura (en inglés) paralela entre el Evangelio Árabe y el Protoevangelio, referente a la estrella de Belén, ver Elliott (2016, p. 137-141)

${ }^{7}$ Para el texto bilingüe, árabe-latín, ver Thilo (1832, p. 70-73), Tischendorf (1853, p. 174).

${ }^{8}$ El pañal, al avanzar en la lectura del relato, asume el rol de una reliquia, y evidencia actos sobrenaturales (Ev. Arab. Inf. 8) (SANTOS OTERO, 2005, p. 142).

${ }^{9}$ Traducción tomada de Santos Otero (2005, p. 142).

${ }^{10}$ Para el texto, en italiano, ver Voicu (2002, p. 21). Nótese que versión larga tiene otro tipo de versificado. Debido a esta diferencia, he insertado entre corchetes la frase "versión larga."
} 
explicando, por ejemplo, que Jesús está una cueva y recostado en un pesebre; y que María y José dialogan con los magos respecto a su lugar de origen, y el propósito de la visita. Al concluir el episodio, la escena del ángel que posee una forma semejante a la estrella que los había llevado hasta la cueva, la que ocurre cinco días después del nacimiento de Jesús, es repetida, conectando ambas versiones en un retrato análogo (Ev. Arab. Inf. 5 [versión larga]).

En consecuencia, en el Evangelio Árabe, así como en el Protoevangelio de Santiago, la estrella guía a los magos al lugar donde Jesús había nacido, convirtiéndola en un astro movible. El Evangelio Árabe, sin embargo, omite describir los pormenores que llevan a los magos, en relación con la estrella, a Belén. El foco pareciera estar en la escena posterior, y que ocurre sólo al finalizar su encuentro con Jesús. Lo significativo de este último episodio comprende la relación de la estrella con un ángel. La forma de la estrella y el ángel aduce semejanza, y realiza una labor idéntica, esto es, dirigir a los magos. Al principio, la estrella los lleva a Jesús. Al concluir, el ángel los conduce a su país de origen. El ángel, señala además el apócrifo, brilla, y es su luz la que los lleva de vuelta a casa. Este detalle evoca la estrella enorme del Protoevangelio, la que resplandecía entre los astros, eclipsándolos, al punto de hacerlos desaparecer (Prot. San. 21.2). En consecuencia, una visión conjunta de ambas versiones del Evangelio Árabe de la Infancia, sugiere que la estrella es de hecho un ángel brillante. Esto explicaría tácitamente, en la visión del Evangelio Árabe, la movilidad del astro.

\section{El Evangelio Armenio de la Infancia}

El Evangelio Armenio de la Infancia puede tentativamente fecharse a finales del siglo sexto (TERIAN, 2008, p. xxvi; PEETERS, 1914, p. lv; PINERO, 2009, p. 300; SANTOS OTERO, 2005, p. 185). Un posible argumento para establecer esta data consiste en vincular el contenido del texto con el movimiento nestoriano que invadió Armenia desde Siria en torno al año 590, y que introdujo apócrifos de la 
vida de Jesús (TERIAN, 2008, p. xix; ELLIOTT, 1993, p. 118; SANTOS OTERO, 2005, p. 185). La validez de esta fecha emerge al notar que un siglo después, el erudito armenio Anania Shirakatsi emplea el apócrifo en uno de sus discursos (CONYBEARE, 1896, p. 323), testificando de este modo de su existencia previa (TERIAN, 2008, p. xix).

Este origen nestoriano implicaría naturalmente que el tal posee rasgos doctrinales inclinados en pos de aquella tradición. Pero la falta de evidencia al respecto ha llevado a cuestionar esta idea (JAMES, 1924, p. 83; GERO, 1971, p. 5253; TERIAN, 2008, p. xix-xxii). Lo cual, con todo, no exime que la procedencia de los misioneros nestorianos, o el texto en si mismo, suponga un arquetipo sirio para el Evangelio Armenio (PEETERS, 1914, p. lv; ELLIOTT, 1993, p. 118; CULLMANN, 2003, p. 457; BURKE, 2017, p. 9). Si bien una opinión de este tipo no está exenta de crítica (GERO, 1971, p. 52-53), el origen sirio del apócrifo, y la posible traducción del mismo desde esta lengua al armenio, cobra fuerza al examinar los rasgos sintácticos del texto, y el uso que este hace de material originario de aquella región (TERIAN, 2008, p. xii-Xxv).

En general, existen dos recensiones en armenio del texto apócrifo, una extensa y una breve (TERIAN, 2008, p. xi, xxvi). No obstante, al establecer esta distinción basada en el tamaño del texto, existe el riesgo de simplificar excesivamente el problema de la recepción textual del Evangelio Armenio. Los manuscritos existentes son numerosos y variados (TERIAN, 2008, p. xxvi-xxx; GEERARD, 1992, p. 40; ELLIOTT, 1993, p. 118), y, en base a la extensión de una u otra recensión, estos presentan diferencias en la escenificación y descripción de la narrativa.

La versión extensa consta de treinta y siete capítulos. La breve, por otro lado, comprende veintiocho (TERIAN, 2008, p. xxvi). Sin embargo, al considerar la historia de los magos y la estrella, el patrón argumental, en ambas versiones, 
comprende relaciones semejantes, y revela el uso que el apócrifo realiza de las fuentes que sirvieron en la composición del documento. ${ }^{11}$

Independientemente de la versión, el autor o editor del Evangelio Armenio empleó como base el Protoevangelio de Santiago y el Evangelio de la Infancia de Tomás (JAMES, 1924, p. 83; ELLIOTT, 2015, p. 271, 286), así como porciones del Evangelio Árabe (ELLIOTT, 1993, p. 118). A este respecto, probablemente, desde el capítulo ocho en adelante, el Evangelio Armenio tomó prestado material del Evangelio Árabe, aunque de un modo bastante libre (PEETERS, 1914, p. xxxxxxiii). La escena de los magos y la estrella de Belén está ubicada dentro de esta sección. Con todo, el Evangelio Armenio edita el relato árabe, el que como se estudió en el apartado anterior, al mismo tiempo construye su contenido en virtud del Protoevangelio. Con todo, el Evangelio Armenio incluye detalles extensos que están ausentes en estos dos últimos textos. ${ }^{12}$

\subsection{La estrella de Belén en el Evangelio Armenio de la Infancia}

El Evangelio Armenio afirma que al concebir María un ángel va al país de los persas para informarles a los magos que deben ir a adorar al niño. Por nueve meses los magos, quienes son reyes, y hermanos, y llevan por nombre Melkon, Gaspar y Baltasar, emprenden un viaje guiados por la estrella hacia Jerusalén (Ev. Arm. Inf. 5.10). ${ }^{13}$ Tres días después del nacimiento de Jesús, los magos arriban a Jerusalén, acompañados de un séquito numeroso de personas. Al llegar a la ciudad, la estrella, la cual los había guiado hasta ahí oculta su brillo, por lo cual se sienten obligados a detener su marcha, y deciden acampar alrededor de Jerusalén. Entonces Herodes, al ver la ciudad rodeada de uma multitud de extranjeros se

\footnotetext{
${ }^{11}$ Esto queda en evidencia al considerar la reconstrucción del Evangelio Armenio realizada por Abraham Terian (2008). Para otros textos ver la traducción al francés de Peeters (1914, p. 69-286), y una selección de textos en español por Santos Otero (2005, p. 186191), y otra completa llevada a cabo por González-Blanco (2015, p. 145-278).

${ }^{12}$ Para una lectura (en inglés) paralela entre el Evangelio Armenio, el Evangelio Árabe y el Protoevangelio, referente a la estrella de Belén, ver Elliott (2016, p. 137-141).

${ }^{13}$ Para el texto, en inglés, ver Terian (2008, p. 48-54). Para el texto en castellano y francés, ver González-Blanco (2015, p. 161) y Peeters (1914, p. 97-98). Santos Otero (2005, p. 187-188) sólo preserva parte de la perícopa.
} 
espanta, y hace llamar a sus consejeros, quienes persuaden al rey enviar un grupo de delegados para conversar con ellos (11.1-5).14

En la entrevista con los enviados del rey, los magos señalan que ellos habían llegado ahí conducidos por un astro. Este iba delante de ellos, y se detenía sobre sus cabezas cuando completaban las diferentes etapas del viaje. Lo significativo de esta declaración estriba en que los magos añaden que, al momento de avanzar, la estrella a veces quedaba a sus espaldas, pero esta tomaba rápidamente la delantera, y se ubicaba en frente (11.8). ${ }^{15}$ Los magos describen otra vez el estado móvil del astro al conversar con Herodes, quien los había convocado para dialogar con ellos cara a cara.

El Evangelio Armenio indica que Herodes intenta impedir el viaje de los magos, pero eventos sobrenaturales ocurren en el palacio, lo cual obliga al rey a dejarlos partir, no sin antes informarles que el Cristo nacería en Belén. Empero, al salir los magos para continuar su viaje, la estrella nuevamente aparece, y los guía hasta la cueva donde estaba Jesús, posándose sobre ella (11.9-14).16 Al concluir la historia, un Jesús que ahora es un niño tiene un encuentro con uno de los soldados que habían venido, junto a los magos, a Belén para visitarlo. El soldado asegura que fueron conducidos por una estrella hasta el lugar donde estaba Jesús (28.11).17

En consecuencia, el Evangelio Armenio delinea el accionar de la estrella en términos de movimiento. Avanza sobre los magos, y procura ir delante de ellos. El aspecto movible asoma aun mas al notar que en tanto los magos adelantan sus pasos, la estrella a fin de no quedar atrás, se posesiona nuevamente delante de ellos. La función del astro consiste en llevar a los magos a Jesús, lo cual se ratifica cuando esta se posa encima de la cueva. Esta acción evoca el Mateo canónico y al

\footnotetext{
${ }^{14}$ Terian (2008, p. 48-50). Para una lectura selectiva, y resumida, ver Elliott (2016, p. 137-138, 140, 144), quien sigue la versión crítica de Terian. Tanto Peeters (1914, p. 131-134), como González-Blanco (2015, p. 181-183), presentan un texto menos crítico, y que se distancia de Terian respecto al tamaño de algunos diálogos. No obstante, el tema de la estrella permanece como una constante estable. ${ }^{15}$ Una variante textual del texto añade un diálogo un tanto mayor entre los magos y los emisarios de Herodes. Con todo, el relato y la descripción de la estrella continúa siendo el mismo; ver Terian (2008, p. 50-51). Consultar Peeters (1914, p. 136), González-Blanco (2015, p. 184).

${ }^{16}$ Terian (2008, p. 51-54). Consultar Peeters (1914, p. 136-141), González-Blanco (2015, p. 184-186).

${ }^{17}$ Terian (2008, p. 126). Consultar Peeters (1914, p. 285), González-Blanco (2015, p. 277$)$.
} 
Protoevangelio, los que estructuran esta parte del relato de una forma semejante (Mt 2,9; Prot. San. 21.3). Un detalle que recuerda el Evangelio Árabe ocurre al observar que un ángel tiene la misión de informar a los magos acerca del nacimiento del Cristo. Si bien el relato no vincula al ángel con la estrella, el Evangelio Armenio parece editar a su contraparte árabe, enfocando el rol e identificación del ángel al principio del episodio, no al final.

\section{El Evangelio de Seudo-Mateo}

El Evangelio de Seudo-Mateo consiste en una adaptación al latín del texto griego del Protoevangelio de Santiago (KAESTLI, 1996, p. 97-99; AMANN, 1910, p. 106; DE STRYCKER, 1965, p. 367), así como probablemente de otros manuscritos en latín que existían de esta última obra, y que hoy están perdidos (EHRMAN; PLEŠE, 2011, p. 73). Otras fuentes pudieron también haber servido de base al Evangelio de Seudo Mateo, tales como el Evangelio de la Infancia de Tomas, y otros relatos apócrifos de Jesús (GIJSEL, 2002, p. 274-275; SANTOS OTERO, 2005, p. 75; KAESTLI, 1996, p. 99). Ahora bien, aunque el Seudo-Mateo presenta una concomitancia literaria directa con el Protoevangelio, este también exhibe diferencias palpables en énfasis y contenido en relación con este último, configurando un propósito y narrativa propia (EHRMAN; PLEŠE, 2011, p. 73; CLAYTON, 2004, p. 19-22).

Uno de esos acentos comprende observar una característica monacal y de castidad en las escenas en que aparece María, estructurando tal accionar como un estilo de vida que ella opta seguir (Ps.-Mt. 7.1-2). Este detalle, en opinión de algunos, parece sugerir que el autor del Seudo-Mateo estaba imbuido de la regla benedictina, un precepto reglamentario monástico que Benito de Nursia escribió en el siglo sexto (AMANN, 1910, p. 106; ELLIOTT, 2015, p. 286). Esta fecha, en base al argumento esgrimido, serviría de base para datar la redacción del apócrifo en este mismo siglo, pues reflejaría una línea narrativa monástica preconcebida en virtud de este documento (AMANN, 1910, p. 106; SANTOS OTERO, 2005, p. 75-76). 
No obstante, si bien existe un elemento lógico al argumentar que la regla de San Benito pudo haber influido en la redacción del Seudo-Mateo en el siglo sexto, también es cierto que esta influencia pudo ocurrir en una fecha posterior, y no necesariamente en paralelo al surgimiento del orden monacal benedictino (EHRMAN; PLEŠE, 2011, p. 75). Examinar el impacto de esta regla en la conformación del apócrifo, con todo, no carece de valor. El problema radica en determinar inapelablemente que el origen del Seudo-Mateo acontece en una fecha equidistante al surgimiento de la vida monacal. Subsanar esta afirmación, proponiendo que su composición parece materializarse una centuria después, cobra una fuerza mayor. En este sentido, una data levemente posterior apela al progreso e influencia paulatina de las ideas, basando sus postulados en el avance de la vida monástica en occidente, y no en sus inicios. A este respecto, el apócrifo pudo haberse escrito en el siglo séptimo, esto es, luego que las órdenes religiosas monásticas comenzaron a expandirse (KLAUCK, 2003, p. 78; KAESTLI, 1996, p. 99-100).

Sin embargo, una opinión tal no está exenta de críticas. Esto, porque el estilo de vida monástico adoptado por María en el evangelio apócrifo no necesariamente alude a una regla monacal. Este retrato, que el autor tal vez habría tomado de la obra Sobre la Virginidad, de Ambrosio de Milán (Virg. 2.6-19), ${ }^{18}$ simplemente comprendería un recurso literario usado para presentar a María como una joven virgen que vive en el templo conforme a las descripciones literarias de la vida ascética femenina del medioevo (BEYERS, 2011, p. 78-83; BEYERS, 2012, p. 129130). Visto así, el autor del Seudo-Mateo acondicionó conceptos propios de su época en un relato en que la virginidad de María cumpliría una función ejemplar.

La consecuencia inmediata de lo antes visto, revela la fragilidad del criterio monacal respecto a la datación del apócrifo (BEYERS, 2011, p. 83). Empero, a pesar de esta limitación, resulta propio reconocer que la tradición textual del SeudoMateo envuelve que el tal no pudo escribirse más allá del siglo nueve. Dos

${ }^{18}$ Para leer el texto ver Schaff; Wace (1983, p. 374-376). 
manuscritos fechados en torno a esta época, el Clmae 316 y el Add. 11880 (BISCHOFF; EBERSPERGER, 1998, v.1, p. 165 [Nr. 759]; v.2, p. 95 [Nr. 2354]), ratifican aquello (BEYERS, 2012, p. 128; BOCKMUEHL, 2017, p. 82; EHRMAN; PLEŠE, 2011, p. 75). Se debe agregar, al mismo tiempo, que existe la posibilidad que un escrito compuesto en el siglo séptimo muestre rasgos puntuales de una relación literaria con el apócrifo, lo cual indicaría que el Seudo-Mateo, o al menos su núcleo primitivo, pudo tal vez surgir alrededor de este tiempo (BERTHOLD, 1989, p. 247-249).

El Evangelio de Seudo Mateo consta de veinticuatro capítulos, siendo los primeros diecisiete una adaptación y expansión del Protoevangelio de Santiago (KLAUCK, 2003, p. 78; KAESTLI, 1996, p. 96-97). Es en el capítulo dieciséis en donde el episodio de los magos y la estrella acontece. Como se verá, aunque el Seudo-Mateo usa como hoja de ruta al Protoevangelio, este omite y añade detalles que vale la pena examinar. ${ }^{19}$ Estos elementos peculiares exponen una riqueza importante de testigos (GIJSEL, 2002, p. 273-300), y por ende variantes textuales (PHILIPPART, 1984, p. 121-131). No obstante, el Seudo-Mateo, a pesar de las diferencias naturales que existen en la transmisión de un texto, el tal desvela escenas en general semejantes. ${ }^{20}$

\subsection{La estrella de Belén en el Evangelio de Seudo-Mateo}

En contraste con el Protoevangelio de Santiago (Prot. San. 18.1; 19.1-3; 21.13), el Seudo Mateo inicia la escena de los magos ubicándolos cronológicamente dos años después del nacimiento de Jesús (Ps.-Mt. 16.1; 17.1). ${ }^{21}$ Estos, informa el relato, traen regalos y llegan a Jerusalén preguntando por el rey que había nacido. Haciendo eco del evangelio canónico de Mateo, los magos dicen haber visto su

\footnotetext{
${ }^{19}$ Para una lectura (en inglés) paralela entre el Protoevangelio de Santiago y el Seudo-Mateo, referente a la estrella de Belén, ver Elliott (2016, p. 137-141)

${ }^{20}$ Nótese esta visión homogénea, al menos en el núcleo narrativo de los capítulos 1 al 17, en los aparatos críticos de los textos modernos que han reconstruido el apócrifo a partir de sus fuentes en latín (EHRMAN; PLEŠE, 2011; TISCHENDORF, 1853). En este trabajo, sigo el texto latino de Ehrman e Pleše (2011, p. 78-112).

${ }^{21}$ Para el texto, en inglés y/o castellano, ver Ehrman e Pleše (2011, p. 102-105), Santos Otero (2005, p. 187-188), González-Blanco (2015, p. 50-51).
} 
estrella en el oriente, y vienen a adorarle (Ps.-Mt. 16.1; Mt 2,2). Herodes se entera y atemoriza, convocando a los líderes religiosos, a quienes les pregunta donde el Cristo nacería de acuerdo a los profetas. Los líderes responden en Belén, y aluden en su totalidad a un oráculo de la Biblia Hebrea citada también por el Mateo canónico (Ps.-Mt. 16.1; Mt 2,6. cf. Miq 5:2). Entonces, como en el Protoevangelio, Herodes hace llamar a los magos, interrogándolos acerca del tiempo que la estrella había aparecido. Una vez que Herodes recibe el reporte, despide a los magos, animándolos a ir a Belén, no sin antes persuadirlos a volver y comunicarle lo que han visto, para que él también vaya y adore a Jesús (Ps.-Mt. 16.1; Prot. San. 21.1; Mt 2,7-8).

Al salir, y en tanto los magos avanzan en su trayecto, el Seudo-Mateo indica que la estrella aparece otra vez (Ps.-Mt. 16.2). En este caso específico, el apócrifo añade que la estrella los precedía, actuando como un guía (ducatus). ${ }^{22} \mathrm{El}$ movimiento de la estrella queda en evidencia, cuando el autor del apócrifo afirma que esta los conduce hasta llegar al lugar donde estaba el niño (Ps.-Mt. 16.2). En tanto el Protoevangelio señala que al arribo de los magos Jesús está en una cueva junto a sus padres, el Seudo-Mateo, por su parte, omite referirse a un lugar (Ps.-Mt. 16.2; Prot. San. 21.2-3).23 En lugar de esto, este dice que al ver la estrella los magos se alegraron, y entran, sin mencionar donde, y ven a Jesús en la falda de María (Ps.-Mt. 16.2). ${ }^{24}$

En consecuencia, en el Seudo-Mateo, así como en el Protoevangelio, el Evangelio Árabe, y el Evangelio Armenio, la estrella no tiene un carácter inmóvil. Antes bien, como el apócrifo lo declara, esta encamina a los magos hasta llegar a donde está Jesús. Inicialmente aparece en el oriente, y su aparición informa a los

\footnotetext{
22 "Euntibus autem magis in uia apparuit stella et quasi ducatum praestaret illis antecedebat eos quousque peruenirent ubi erat puer" (Ps.-Mt. 16.2) (EHRMAN; PLEŠE, 2011, p. 104). Por su parte, el texto de Tischendorf (1853, p. 79) dice así: "E untibus autem magis in via apparuit eis stella, quae quasi dux eis erat, antecedens eos quousque pervenirent ubi puer erat." Nótese que, a pesar de las variantes, "ducatum/dux," ambos vocablos, de raíz semejante, apelan entender la estrella como un guía.

${ }^{23}$ El Seudo Mateo, con todo, también afirma que Jesús nació en una cueva (spēlunca), y que luego fue trasladado, junto a su madre, a un establo (stabulum) (Ps.-Mt. 13.2; 14.1) (EHRMAN; PLEŠE, 2011, p. 98, 102; TISCHENDORF, 1853, p. 79). Sin embargo, en el caso de la escena de los magos, no existe un referente espacial que indique donde los magos entraron.

${ }^{24}$ Existe una variante textual que inserta el sustantivo casa (domus), lo cual envolvería que Jesús, como el Protoevangelio indica, estaba en un lugar específico (cueva/casa) junto a sus padres (Prot. San. 21.3). Sin embargo, la variante no tiene el apoyo textual necesario que permita incluirla en el texto en latín. Ver Ehrman e Pleše (2011, p. 104) y Tischendorf (1853, p. 79).
} 
magos el nacimiento de un rey. El objetivo de la aparición comprende conducirlos a Jesús. El texto latino asume esta noción, al indicar semánticamente el rol conductor del astro (ducatus). No existe, empero, indicación que la estrella descienda para mostrar el lugar en el que Jesús está con sus padres, como lo hace el Protoevangelio o el Evangelio Armenio. El desplazamiento del astro, por tanto, ocurre presumiblemente sólo en el cielo, ayudando a que los magos encuentren a Jesús.

\section{Conclusión}

El motivo de la estrella de Belén, que ocurre inicialmente en el Evangelio de Mateo, aparece primero en el Protoevangelio de Santiago. El Protoevangelio edita y emplea las escenas de la estrella en Mateo, y apunta no sólo a su rol de conducción, un acto singular que éste toma prestado de él, sino además a su aspecto y tamaño. Este acto de conducción, que remeda al Mateo canónico, tiene como propósito único anunciar el nacimiento de Jesús, y llevar a los magos hasta su presencia.

El Evangelio Árabe, si bien emula el contenido del Protoevangelio y del Mateo canónico, incluye un detalle angélico, que posiblemente el Evangelio Armenio igualmente imita. En ambos casos, el cuerpo celeste aludiría a un ángel, lo que elucidaría el porque del movimiento del astro. El Evangelio Árabe, además, enfatiza el brillo del ángel, lo cual estaría en paralelo, al menos en relación al resplandor, con el fulgor del astro en el Protoevangelio. En los dos casos, el apócrifo Árabe y Armenio, la estrella tiene como objetivo conducir a los magos hacia donde está Jesús.

El Seudo-Mateo también imita el contenido del Protoevangelio, pero en términos breves. Con todo, a pesar de su laconismo narrativo, el Seudo-Mateo deja en claro el aspecto móvil del astro. De hecho, el texto apócrifo subraya esta acción usando un término latino específico que delinea el rol de guía. A la par, como en los tres apócrifos mencionados anteriormente, la finalidad única de la estrella consiste en llevar a los magos a Jesús. 
En general, los cuatro evangelios apócrifos investigados proceden tanto del sector oriental (Protoevangelio, Evangelio Árabe, Evangelio Armenio) como del occidental (Pseudo-Mateo). La distribución geográfica de estos documentos, parece sugerir una expansión en el planteamiento de las ideas, o la preservación de tradiciones. Esto queda aun más en evidencia al notar las diversas lenguas en las que estos textos fueron originalmente escritos, preservados y/o traducidos (griego, siriaco, armenio, árabe, latín). Esta variedad lingüística y de propagación territorial acusa una interpretación que podría sugerir una visión permanente respecto al rol literario de la estrella en los siete primeros siglos. En particular en los documentos que fueron compuestos a partir del Protoevangelio de Santiago.

A este respecto, si bien existen cambios editoriales, y embellecimientos en torno a la figura de los magos, y los hechos del nacimiento de Jesús, el motivo de la estrella permanece, en esencia, semejante. El atributo común que surge de estos cuatro relatos es el rasgo movedizo que la estrella posee. El astro de Belén, sea este un cuerpo celeste sobrenatural o un ángel, tiene la facultad de avanzar, desaparecer y detenerse a medida que las circunstancias lo requieran. $\mathrm{Al}$ mismo tiempo, la meta del astro, en todas las narraciones tiene que ver con Jesús, y el aspecto de conducción sobrenatural que esta adquiere. En este sentido, los cuatro evangelios apócrifos concuerdan, en general, con la descripción de la estrella del Mateo canónico, el que también retrata el papel y las particularidades del astro de un modo análogo.

\section{REFERENCIAS}

ALLISON, D. C. The Magi's Angel (Matt. 2:2, 9-10). In: Allison, D. C. (ed.). Studies in Matthew: interpretation past and present. Grand Rapids: Baker Academic, 2005. p. 17-41. AMANN, E. Le protévangile de Jacques et ses remaniements latins: introduction, textes, traduction et commentaire. Paris: Letouzey et Ané, 1910.

BARTHEL, P.; VAN KOOTEN, G. H. (ed.). The Star of Bethlehem and the Magi: interdisciplinary perspectives from experts on the Ancient Near East, the Greco-Roman World, and Modern Astronomy. Leiden: Brill, 2015. 
BAUER, W. et al. A Greek-English of the New Testament and other Early Christian literature. Chicago: University of Chicago Press, 2000.

BERTHOLD, M. Zur datierung des Pseudo-Matthäus-Evangeliums. Wiener Studien, v. 102, p. 247-249, 1989.

BEYERS, R. La règle de Marie: caractère littéraire et inspiration monastique. Apocrypha, v. 22, p. 49-86, 2011.

BEYERS, R. The transmission of Marian Apocrypha in the Latin Middle Ages.

Apocrypha, v. 23, p. 117-140, 2012.

BISCHOFF, B.; EBERSPERGER, B. Katalog der festländischen Handschriften des neunten Jahrhunderts. Wiesbaden: Harrassowitz, 1998. 4 v. (mit Ausnahme der wisigotischen).

BOCKMUEHL, M. Ancient apocryphal gospels. Louisville: Westminster/John Knox Press, 2017.

BUDGE, E. A. W. Legends of our Lady Mary the Perpetual Virgin and her mother Hanna. London: Medici Society, 1922.

BURKE, T. The Syriac Tradition of the Infancy Gospel of Thomas: a critical edition and english translation. Piscataway, NJ: Gorgias Press, 2017.

CARTLIDGE, D. R. How to draw an immaculate conception: Protevangelium Jacobi 11-12 in Early Christian art. In: DOBLE, P.; KLOHA, J. (ed.). Texts and traditions: essays in honour of J. Keith Elliott. Leiden: Brill, 2014. p. 319-326.

CARTLIDGE, D. R.; ELLIOTT, J. K. Art and the Christian apocrypha. London: Routledge, 2001.

CLAYTON, M. The Apocryphal Gospels of Mary in Anglo-Saxon England. Cambridge: Cambridge University Press, 2004.

CONYBEARE, F. C. Ananias of Shirak upon Christmas. The Expositor, v. 5, n. 4, p. 321$337,1896$.

COTHENET, E. Le Protévangile de Jacques: origine, genre et signification d'un premier midrash chrétien sur la Nativité de Marie. Aufstieg und Niedergang der Römischen Welt. Band 2, v. 25, n. 6, p. 4252-4269, 1988.

COWPER, B. H. The Apocryphal Gospels and other documents relating to the history of Christ. London: Williams and Norgate, 1867.

CULLMANN, O. Infancy Gospels. In: SCHNEEMELCHER, W. (ed.). New Testament apocrypha: Gospels and related writings. rev. Louisville: Westminster: John Knox Press, 2003. v. 1. p. 414-469.

DE ALDAMA, J. A. Fragmentos de una versión latina del Protoevangelio de Santiago y una nueva adaptación de sus primeros capítulos. Biblica, v. 43, n. 1, p. 57-74, 1962. 
DE ALDAMA, J. A. Un nuevo testigo indirecto del Protoevangelio de Santiago. In:

LIVINGSTONE, E. A. (ed.). Studia patristica. Berlin: Akademie Verlag, 1975. p. 79-82.

DE STRYCKER, É. Une ancienne version latine du Protévangile de Jacques avec des extraits de la Vulgate de Matthieu 1-2 et Luc 1-2. Analecta Bollandiana, v. 83, n. 3-4, p. 365-410, 1965 .

DUCHESNE-GUILLEMIN, J. The wise men from the East in the western tradition. Acta Iranica, v. 10, n. 24, p. 149-157, 1985.

EHRMAN, B. D.; PLEŠE, Z. The apocryphal gospels: texts and translations. New York: Oxford University Press, 2011.

EL CORÁN. Versión al español de Juan B. Bergua. Madrid: Ediciones Ibéricas, 2007.

ELLIOTT, J. K. The apocryphal New Testament: a collection of apocryphal christian literature in an english translation. Oxford: Clarendon Press, 1993.

ELLIOTT, J. K. Christian apocrypha and the developing role of Mary. In: GREGORY, A. et al. (ed.). The Oxford Handbook of early Christian apocrypha. Oxford: Oxford University Press, 2015. p. 269-288.

ELLIOTT, J. K. A synopsis of the apocryphal nativity and infancy narratives. Leiden: Brill, 2016.

FERRARI-D'OCCHIEPPO, K. The Star of the Magi and Babylonian Astronomy. In: VARDAMAN, J.; YAMAUCH, E. M. (eds.). Chronos, Kairos, Christos: nativity and chronological studies presented to Jack Finegan. Winona Lake: Eisenbrauns, 1989. p. 4153 .

FOSTER, P. The Protevangelium of James. Expository Times, v. 118, n. 12, p. 573-582, 2007.

FOSTER, P. The Protevangelium of James. In: FOSTER, P. (ed.). The non-canonical gospels. London: Bloomsbury T. \& T. Clark, 2008. p. 110-125.

GEERARD, M. Clavis apocryphorum Novi Testamenti. Turnhout: Brepols, 1992.

GERO, S. The infancy gospel of Thomas: a study of the textual and literary problems.

Novum Testamentum, v. 13, n. 1, p. 46-80, 1971.

GIJSEL, J. Nouveaux témoins du Pseudo-Matthieu. Sacris Erudiri, v. 41, p. 273-300, 2002.

GILES, J. A. Codex apocryphus Novi Testamenti: the uncanonical gospels and other writings. Part 1. London: D. Nut, 1852.

GONZÁleZ, J.; ISART, C.; GONZÁLEZ, P. El Protoevangelio de Santiago. Madrid: Ciudad Nueva, 1997.

GONZÁLEZ-BLANCO, E. Evangelios apócrifos. Valladolid: MAXTOR, 2015. 
GOODACRE, M. The Protevangelium of James and the creative rewriting of Matthew and Luke. In: WATSON, F.; PARKHOUSE, S. (ed.). Connecting gospels: beyond the canonical/non-canonical divide. Oxford: Oxford University Press, 2018. p. 57-76.

GRYPEOU, E. The table from heaven: a note on Qur'an, Surah 5,111 Ff. Collectanea Christiana Orientalia, v. 2, p. 311-316, 2005.

HEGEDUS, T. The Magi and the Star in the Gospel of Matthew and Early Christian Tradition. Laval Théologique et Philosophique, v. 59, n. 1, p. 81-95, 2003.

HORN, C. Intersections: the reception history of the protoevangelium of James in sources from the Christian East and in the Qu'Rān. Apocrypha, v. 17, p. 113-150, 2006.

HORN, C. Mary Between bible and Qur'an: soundings into the transmission and reception history of the protoevangelium of James on the basis of selected literary sources in Coptic and Copto-Arabic and of Art-historical Evidence pertaining to Egypt. Islam and Christian-Muslim Relations, v. 18, n. 4, p. 509-538, 2007.

HORN, C. Syriac and Arabic Perspectives on Structural and Motif Parallels Regarding Jesus' Childhood in Christian Apocrypha and Early Islamic Literature: the 'Book of Mary', the Arabic Apocryphal Gospel of John and the Qur'ān. Apocrypha, v. 19, p. 267-291, 2008.

JAMES, M. R. The Apocryphal New Testament. Oxford: The Clarendon Press, 1924.

JÖRG, F. Texts about Jesus: non-canonical gospels and related literature. In: GREGORY, A. et al. (ed.). The Oxford handbook of early Christian apocrypha. Oxford: Oxford University Press, 2015. p. 13-47.

KAESTLI, J. D. Le protévangile de Jacques en latin. État de la question et perspectives nouvelles. Revue d'Histoire des Textes, v. 26, p. 41-102, 1996.

KAESTLI, J. D. Le protévangile de Jacques latin dans l'homélie inquerendum est pour la fête de la nativité de Marie. Apocrypha, v. 12, p. 99-154, 2001.

KLAUCK, H. J. Apocryphal gospels: an introduction. London: T. \& T. Clark International, 2003.

LIDDELL, H. G.; SCOTT, R. A Greek-English lexicon. Oxford: The Clarendon Press, 1996.

PEETERS, P. A propos de l'Évangile arabe de l'Enfance. Le manuscrit de J. Golius. Analecta Bollandiana, v. 41, p. 132-134, 1923.

PEETERS, P. Évangiles apocryphes II: l'Évangile de l’Énfance. Paris: Auguste Picard, 1914 .

PHILIPPART, G. Le Pseudo-Matthieu au risque de la critique textuelle. Scriptorium, v. 38, p. 121-131, 1984.

PIÑERO, A. (ed.). Todos los evangelios: canónicos y apócrifos. Madrid: Edaf, 2009. 
QUARLES, C. L. The Protevangelium of James as an Alleged Parallel to Creative Historiography in the Synoptic Birth Narratives. Bulletin for Biblical Research, v. 8, p. 139-149, 1998.

ROBERTS, A.; DONALDSON, J. The Ante-Nicene Fathers: translations of the Writings of the Fathers down to A.D. 325. Grand Rapids: Eerdmanns, 1989. 10 v.

SANTOS OTERO, A. D. Los evangelios apócrifos. Madrid: Biblioteca de Autores Cristianos, 2005.

SCHAFF, P.; WACE, H. A select library of Nicene and Post-Nicene Fathers of the Christian Church. Grand Rapids: Eerdmans, 1983. 14 v.

SCHNEEMELCHER, W. General introduction. In: SCHNEEMELCHER, W. (ed.). New Testament apocrypha: gospels and related writings. Louisville: Westminster: John Knox Press, 2003. v. 1. p. 9-75.

SCHRÖTER, J. The formation of the New Testament canon and early Christian apocrypha. In: GREGORY, A. et al. (ed.). The Oxford handbook of early Christian apocrypha. Oxford: Oxford University Press, 2015. p. 167-184.

SIKE, H. Evangelium infantiae, vel Liber apocryphus de infantia Servatoris ex manuscripto edidit, ac latina versione et notis illustravit. Trajecti ad Rhenum [Utrecht]: Franciscum Halmam, 1697.

TERIAN, A. The Armenian Gospel of the Infancy. Oxford: Oxford University Press, 2008.

THILO, J. K. Codex Apocryphus Novi Testamenti. Leipzig: Vogel, 1832. v. 1.

TISCHENDORF, C. V. Evangelia Apocrypha. Lipsiae: Avenarjus et Mendelssohn, 1853.

VIVIANO, B. T. The movement of the star, Matt 2: 9 and Num 9: 17. Revue Biblique, v. 103, n. 1, p. 58-64, 1996.

VOICU, S. J. Vangelo arabo dell'infanzia di Gesù. Roma: Città Nuova, 2002.

ZERVOS, G. T. The Protevangelium of James and the composition of the Bodmer miscellaneous codex: chronology, theology, and liturgy. In: MCDONALD, L. M.; CHARLESWORTH, J. H. (ed.). 'Non-canonical' religious texts in Early Judaism and Early Christianity. London: T. \& T. Clark, 2012. p. 177-194. 